\title{
Numerical Simulation on the Effects of Component Layout Orientation on the Performance of a Neon-Charged Cryogenic Loop Heat Pipe
}

\author{
Fa-Long $\mathrm{He}^{1,4} \cdot$ Wang-Fang Du ${ }^{1,3} \cdot$ Jian-Fu Zhao ${ }^{1,3}$ (D) $\cdot$ Jin-Dong $\mathrm{Li}^{2} \cdot$ Jian-Yin Miao ${ }^{2} \cdot$ Jiang $\mathrm{He}^{2}$
}

Received: 26 June 2019 / Accepted: 22 October 2019 / Published online: 11 December 2019

(C) Springer Nature B.V. 2019

\begin{abstract}
As a typically highly efficient two-phase heat transfer under cryogenic temperature range, the operation performances of cryogenic loop heat pipes (CLHPs) might be affected by the relative location of different components. A transient mathematical model is established in present work. The model validation is demonstrated by comparing the simulation results with an auxiliary loop type neon-charged CLHP (Ne-CLHP) experiment data, and a good agreement is achieved. The effect of gravity caused by different layout orientations on the Ne-CLHP operation performances are then investigated numerically. There are three operation modes, namely normal LHP mode (n-LHP), gravity-assisted LHP mode (g-LHP), and gravity thermosyphon mode (GTP), according to different layout orientations and heat loads. Under the gravity resistance condition with a positive layout inclination angle, the Ne-CLHP is operated in n-LHP. The operating temperature increases with the positive layout inclination angle, and the heat transport capacity decreases. Under the gravity assistance condition with a negative layout inclination angle, all three operation modes may occur according mainly to the primary heat load. Only the two LHP modes are simulated in the present study, which located in the range between the transition heat load from GTP to g-LHP and the heat transport capacity. The lager negative layout incline angle, the higher transition heat load and the heat transport capacity, while lower operating temperature. In addition, the gravity-independence of cross-sectional two-phase distribution in the transport lines is discussed in the frame of dominant force analysis. The modeling effort will contribute to the technology research and development, as well as the operation control of CLHPs for space and ground applications.
\end{abstract}

Keywords Cryogenic loop heat pipe $\cdot$ Transient model $\cdot$ Layout orientation $\cdot$ Operation performance $\cdot$ Gravity effect

This article belongs to the Topical Collection: Multiphase Fluid Dynamics in Microgravity

Guest Editors: Tatyana P. Lyubimova, Jian-Fu Zhao

Jian-Fu Zhao

jfzhao@imech.ac.cn

$\triangle$ Jin-Dong Li

ljdcast@163.com

1 CAS Key Laboratory of Microgravity, Institute of Mechanics, Chinese Academy of Sciences, Beijing 100190, China

2 Beijing Key Laboratory of Space Thermal Control Technology, Institute of Spacecraft System Engineering, China Academy of Space Technology, Beijing 100094, China

3 School of Engineering Science, University of Chinese Academy of Sciences, Beijing 100049, China

4 School of Energy and Safety Engineering, Tianjin Chengjian University, Tianjin 300384, China

\section{Introduction}

Cryogenic loop heat pipe (CLHP) is a kind of loop heat pipes (LHPs) operating in cryogenic temperature range, which is developed particularly for satisfying the requirements of efficient thermal link between cryogenic heat sources, such as infrared radiation (IR) sensors/detectors, super-conducting magnet and small-scale particle detectors, and cryocoolers to meet the increasing demands of cryocooler-based space applications. Its development was initiated at the beginning of this century, and considerable achievement has been obtained (Bai et al. 2015b). Generally, cryogenic fluids are in supercritical state at room temperature, and have small surface tension and low latent heat of vaporization, resulting in difficulty in the supercritical start-up, as well as weak driving force and then low heat transport capability and weak operation 
stability. Furthermore, the huge temperature difference between the working fluid and the ambient environment also causes leads to serious parasitic heat loss, which strengthens the weakening of system performance. Up to now, several types of CLHPs with different system structures and operating temperatures have been developed to realize the supercritical start-up and stable operation in corresponding cryogenic temperature range. According to Bai et al. (2015b), an auxiliary loop type CLHP, which has an additional auxiliary loop composed of a secondary evaporator, secondary compensation chamber, secondary condenser, secondary liquid and vapor lines in addition to the gas reservoir, is effective to help realize the supercritical start-up of CLHPs, and can be applicable in both ground and space conditions. The auxiliary loop, however, brings added complexities in the loop. The present study focuses on this type of CLHP, particularly that with neon as the working fluid, or auxiliary loop type neoncharged CLHP (Ne-CLHP).

Yun et al. (2002) firstly reported the experimental test of an auxiliary loop type LHP with ethane as the working fluid for passive optical bench cooling applications. Experimental results clearly demonstrated its capability, i.e. it could start up reliably from a supercritical temperature of $335 \mathrm{~K}$ to achieve a normal operating temperature of $215 \mathrm{~K}$, and achieve a $50 \mathrm{~W}$ heat transport capability with $5 \mathrm{~W}$ applied to the secondary evaporator. The operating temperature range, however, was 215-218 K, not in cryogenic temperature range, and then the studied auxiliary loop type LHP still belongs to the category of ambient loop heat pipes (ALHP). From then on, several cryogenic working fluids are used in different cryogenic temperature ranges, including methane for 100-190 K (Guo et al. 2018b), nitrogen for 80-120 K (Hoang et al. 2003; Bugby et al. 2003; Gully et al. 2011; Zhao et al. 2011; Bai et al. 2012, 2013), hydrogen for 20-30 K (Hoang et al. 2002), and neon for 30-40 K (Bugby et al. 2004; He et al. 2017; Guo et al. 2017, 2018a), respectively. Based on the experimental results, the following important conclusions have been drawn: 1) the CLHP could realize the supercritical startup with suitable heat load applied to the secondary evaporator, and the larger the heat load applied to the secondary evaporator, the sooner the temperature drop process of the primary evaporator; 2) the primary evaporator could operate independently with large heat load, whereas the secondary evaporator must be kept in operation to assist the normal operation of the primary evaporator with small heat load; 3 ) the CLHP had the ability to operate with a small heat load applied to the primary evaporator for a long time, and manifested good thermal control performance; 4) there existed an optimum auxiliary heat load and charged pressure of the working fluid respectively during the supercritical startup.

Comparing with experiment research, theoretical analysis and numerical simulation can also play an important role for understanding the heat transfer performance and the underlying mechanism of both CLHP and ALHP. Quite a few modeling and theoretical studies, however, have been carried out on ALHPs (Kaya et al. 2008; Kaya and Hoang 1999; Kaya et al. 2008; Launay et al. 2008; Nishikawara et al. 2013), while quite limited and obviously inadequate works on CLHP. A steady mathematical model for an auxiliary loop type nitrogen-charged CLHP $\left(\mathrm{N}_{2}\right.$-CLHP) was established by Bai et al. (2010) based on the conservations of mass, momentum and energy of each component, and the modeling results including the heat transport capacity and operating temperature variation trend showed good agreement with the experimental data. Qu et al. (2018) presented a steady global model of CLHP coupled with a finer heat leak model in the evaporator core by full consideration of the subcooled effect and the mass flow rate variation of the working fluid along the evaporator core, which improves the prediction accuracy. To simulate the transient behaviors and supercritical start-up of CLHP, Bai et al. (2011) developed a transient mathematical model with a simplified treatment of the thermodynamic properties of the working fluid to obtain computation efficiency. This simplified treatment, however, may result in great deviation from the real thermodynamic sate of the working fluid, especially for supercritical state due to dramatic changes of thermodynamic properties of the working fluid. Recently, $\mathrm{He}$ et al. (2018) developed a transient mathematical model for CLHP. Ignoring the gravity influence, the basic route of thermodynamic processes and operating characteristics of an auxiliary loop type neon-charged CLHP (Ne-CLHP) were analyzed, corresponding to both the microgravity case for the space applications and the horizontal layout case in normal gravity environment during the ground tests.

The gravity influence, however, may play an important role during the ground tests and ground applications. For example, Chuang et al. (2014) presented an experimental study of an ALHP operating at small positive elevation $(0-127 \mathrm{~mm})$ in terrestrial condition. A unique triple-inflection operating temperature curve was observed. Combined with analytical study, the author categorized the steady state operation modes of ALHP into capillary-controlled and gravity-controlled mode. Similarly, Bai et al. (2015a) identified two driving modes of ALHP under the gravity-assisted attitude: gravity-driven mode and capillarity-gravity co-driven mode, based on the experiments at large positive elevation $(1-2 \mathrm{~m})$. A biinflection operating temperature curve, different from the observations of Chuang et al. (2014), was observed, which may be caused mainly by the difference of positive elevations. There could be similar operating performance of CLHP with an auxiliary loop under gravity assisted condition, which has never been reported in the literature. In order to understand the complicated operating characteristics of CLHPs, especially for terrestrial applications and/or ground tests of such devices developed for space applications (Okamoto et al. 2019), our 
previous transient mathematical model (He et al. 2018) will be used in the present study to simulate the gravity influences on operation performance of an auxiliary loop type Ne-CLHP, particularly focusing on the effects of relative height difference between the primary and secondary compensation chambers.

\section{Mathematical Model}

\section{Basic Hypotheses}

The transient mathematical model developed to simulate the operation performance of an auxiliary loop type Ne-CLHP is based on the node-network method, in which the mass and energy conservation equations are applied to the control volume of fluid nodes, the momentum equation is applied to the fluid flow path between adjacent fluid nodes, and the energy conservation equation is also applied to the wall node (He et al. 2018). The treatment of the fluid flow is thus one dimensional in nature. Furthermore, the follow hypotheses are supposed:

1) The fluid at each fluid node is always in a state of local thermodynamic equilibrium and satisfies the Gibbs phase rule. It means there are only two independent thermodynamic state parameters (such as temperature and density) for single-component fluid, and other parameters are determined by them.

2) The temperature of solid frame of the capillary wick and the fluid flowing through the capillary wick are the same, which means the effects of interfacial thermal contact resistance of vapor-solid and liquid-solid, as well as the thermal inertia of the capillary wick are neglected.

3) The homogenous model is used for the two-phase gasliquid flow and the corresponding two-phase heat transfer, which means the distribution of the vapor and liquid phases is uniform and the velocities of the two phases are the same in each fluid node. It further indicates that the effects of gravity on cross-sectional phase distributions in transport lines and the primary and secondary condensers are ignored.

\section{Governing Equations}

The mass conservation equation for the $i$-th fluid node is

$\frac{d m_{i}}{d t}=\sum_{K} m_{i K}$

where $m_{i}$ denotes the mass of the $i$-th fluid node, $\dot{m}_{i K}$ denotes the mass flow rate between the $i$-th fluid node and its adjacent node (the $K$-th fluid node).

The energy conservation equation for the $i$-th fluid node is

$\frac{d\left(m_{i} h_{i}\right)}{d t}=\sum_{K}\left(m_{i K} h_{i K}\right)+\sum_{M} Q_{i M}+W_{i}$

where $h_{i}$ denotes the specific enthalpy of the $i$-th fluid node, $\dot{m}_{i K} h_{i K}, Q_{i M}$, and $W_{i}$ denote the enthalpy flow rate between the $i$-th fluid node and its adjacent fluid node (the $K$-th fluid node), the heat transfer between the $i$-th fluid node and its adjacent wall node (the $M$-th wall node), the compression work caused by the pressure variation of the $i$-th fluid node. It should be noted that the value of $h_{i K}$ is depended on the direction of $\dot{m}_{i K}$. An upstream scheme is used for the specific enthalpy to favor the fluid flow direction, i.e. the value always be the specific enthalpy of the upstream fluid node corresponding to the $i$-th fluid node.

The energy conservation equation of the $j$-th wall node is

$m_{j} C_{j} \frac{d T_{j}}{d t}=\sum_{N} Q_{j N}$

where $m_{j}$ and $C_{j}$ denote the mass and specific heat capacity of the $j$-th wall node, $Q_{j N}$ denotes the heat exchange between the $j$-th wall node and its adjacent node (the $N$-th node), which might be a wall node, a fluid node, the ambient or the heat load/sink applied to the system.

The momentum equation for the $i-K$ flow path between the $i$-th fluid node and its adjacent one (the $K$-th fluid node) is

$$
\left(\frac{L_{e f f, i K}}{A_{e f f, i K}}\right) \frac{d m_{i K}}{d t}=P_{i}-P_{k}+\Delta P_{a}+\Delta P_{f}+\Delta P_{g}
$$

where $P_{i}$ and $P_{K}$ denote respectively the pressures of the upstream and downstream fluid nodes of the corresponding flow path, $\Delta P_{f}, \Delta P_{a}$, and $\Delta P_{g}$ denote respectively the pressure drops caused by friction, acceleration and gravity, $L_{e f f, i K}$ and $A_{\text {eff,iK }}$ denote the equivalent length and effective flow area.

The capillary wick is wetted by the liquid, and the evaporating menisci is formed and located at its out layer. The governing equation for the phase-change interface is

$\dot{m}_{e v}=\frac{Q_{e v}}{\Delta h}$

where $\dot{m}_{e v}$ and $\Delta h$ denote respectively the mass flow rate from the vapor-liquid phase change interface and the latent heat of vaporization. $Q_{e v}$ denotes the required heat for evaporation, which is determined by the heat transfer from evaporator wall, the heat associated with the working fluid flowing from the compensation chambers, and the heat loss to the compensation chambers from evaporating interface.

The common-used empirical correlations for the single and two-phase flow, as well as heat transfer, are adopted. The detail formulations can be found in He et al. (2018). 
Combined with the physical property database of the working fluid, as well as appropriate boundary and initial conditions, the above equations provide a closed model for transient behaviors of an auxiliary loop type Ne-CLHP.

\section{Solution Method}

The initial conditions include the temperature and quality of fluid and the wall temperature for each component, which is determined by system physical state. Then the routine of property database and common-used empirical correlations is called for calculating the thermal property and heat transfer coefficient. After initialization of the nodes, the boundary conditions are applied, which include system charged pressure, applied heat load, ambient and heat sink temperature. Then, the new step values of density, enthalpy, mass flow rate and wall temperature could be calculated by the governing equations.

The integration method of the Differential Algebraic System SoLver (DASSL) (Brenan et al. 1996) is used to solve the governing equations. The derivatives are approximated by the $k$-th order backward differentiation formula (BDF), where $k$ ranges from one to five. The Newtonian iteration technique is applied to solve next step value. The step size is estimated by the relative and absolute tolerances at each time step to maintain calculation stability and to reduce the number of iterations. Note that, the numerical calculation will continue until the pressure drop of the primary and/or secondary loop is greater than the maximum capillary pressure provided by the capillary wick of the primary and/or secondary evaporators, respectively. Then the limit heat transport capacity can be determined using the way of backward interpolation between the failure stage and its previous stage.

\section{Model Validation}

To validate the model, the simulation results are compared with the experimental data (Guo et al. 2017). The Ne-CLHP structure is shown in Fig. 1, which is composed of a primary evaporator (PE), a primary vapor line (PVL), a primary condenser (PC), a primary liquid line (PLL), a primary compensation chamber (PCC), a secondary liquid line (SLL), a secondary condenser (SC), a secondary compensation chamber (SCC), a secondary evaporator (SE), a secondary vapor line (SVL), and a gas reservoir (GR). The structural parameters of the main components are listed in Table 1, where OD and ID denote the outer and inner diameter, respectively. Most components were made of stainless steel except the capillary wicks inside PE and SE which were made of sintered nickel powders.

The ambient temperature was $296 \mathrm{~K}$, the system charged pressure was $3.19 \mathrm{MPa}$, and the heat sink temperature is controlled by a G-M cryocooler. All simulation conditions are the same as those in the experiments. Since no measurement of the heat sink temperature was made in the experiments, it is set as a function of time related to the measured wall temperature of SCC. SE is applied a constant heat load of $1.5 \mathrm{~W}$. The effective heat transfer coefficient between the condenser line and the heat sink is set as $240 \mathrm{~W} /\left(\mathrm{m}^{2} \cdot \mathrm{K}\right)$, while the effective heat conductance between the evaporator wall and the evaporating interface is set as $5 \mathrm{~W} / \mathrm{K}$.

Figure 2 shows the comparisons of the system pressure $\left(P_{s y s}\right)$ and the operating temperature or the wall temperature of PE $\left(T_{P E}\right)$ between the simulation results and the experimental data. The experimental observations of the fluctuations and trends of the system pressure and operating temperature are well captured by the numerical simulation with a satisfactory agreement. The maximum deviation of the system pressure is about $0.05 \mathrm{MPa}$, and the maximum operating temperature difference occurred at the primary heat load of $4.2 \mathrm{~W}$ is about $0.5 \mathrm{~K}$.

\section{Simulation Results and Analysis}

The layout inclination angle $\theta$ reflects the relative height difference between the primary and secondary compensation chambers (Fig. 3), which results in a gravity pressure drop under gravity resistance condition or a head of gravity under gravity assistance condition in the momentum equation even if no influence of gravity on the cross-sectional phase distribution is taken into account. When the Ne-CLHP is set in the horizontal position $\left(\theta=0^{\circ}\right)$, the gravity pressure drop or the head of gravity is absent. When $\theta<0$, the working fluid flows through PLL in the same direction of gravity or its longitudinal component and then gravity is an assistant or driving force, while the flow direction in PVL and SLL are opposite and gravity acts as a resistant force. Otherwise, the opposite is true.

Figure 4 shows the variations of primary loop pressure drop with the primary heat load under different layout inclination angle. Here, the heat sink temperature is set as a constant of $30 \mathrm{~K}$. The ambient temperature is $296 \mathrm{~K}$. The system charged pressure of the working fluid is $3.19 \mathrm{MPa}$. The total parasitic heat loss is set as $0.25 \mathrm{~W}$, which is distributed uniformly on all nodes of the transport lines. The secondary heat load applied on SE is set constantly as $0.5 \mathrm{~W}$, while the primary heat load applied on PE is increased step-by-step from $0.3 \mathrm{~W}$ with a step value of $0.1 \mathrm{~W}$. It lasts for $4000 \mathrm{~s}$ in the numerical simulation for each step of the primary heat load in order to obtain a steady state of the operation. The total pressure drop of the primary loop $\left(\Delta P_{P L}\right)$ increases with the increase of the primary heat load for a fixed layout inclination angle. On the other hand, the maximum capillary pressure $\left(\Delta P_{\text {Max.Cap }}\right)$ can be calculated by the surface tension and minimum effective radius of the capillary wick using Young- 


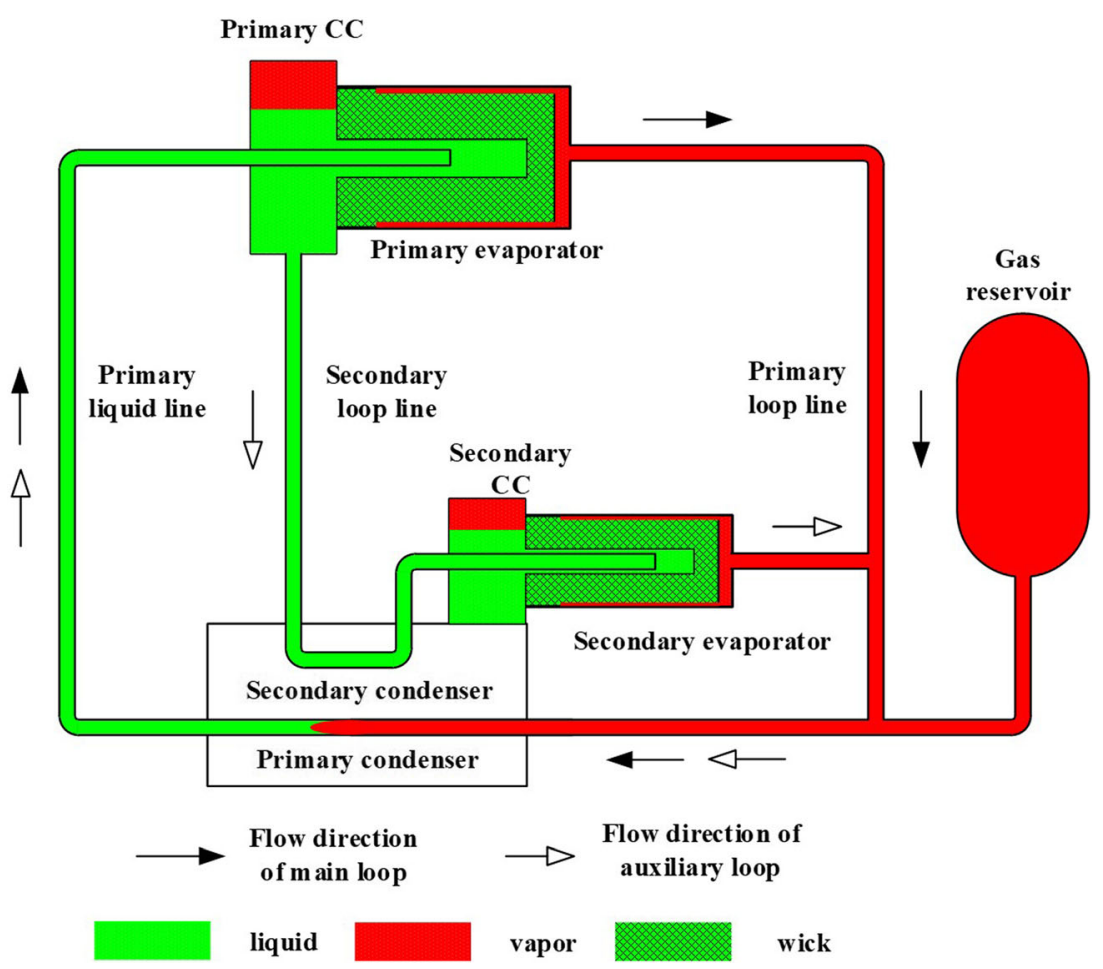

Fig. 1 Schematic view of the Ne-CLHP

Laplace equation. The maximum capillary pressure is decreasing with the primary heat load due to the increasing operating temperature and corresponding fluid temperature at the evaporating interface. When the pressure drops of the primary and/ or secondary loops reach the maximum capillary pressure, the $\mathrm{Ne}$-CLHP will reach the capillary-driven limit. Thus, the heat transport capacity $\left(Q_{\text {Max.PE }}\right)$ can be determined by using a backward interpolation between the first failure stage, in which the pressure drop of the primary or secondary loops exceeds for the first time the maximum capillary pressures provided respectively by the capillary wicks of the primary

Table 1 Basic parameters of the Ne-CLHP

\begin{tabular}{lll}
\hline Components & Parameters & Dimensions \\
\hline Primary evaporator & Casing OD/ID $\times$ length $/ \mathrm{mm}$ & $13 / 11 \times 50$ \\
& Wick OD/ID $\times$ length $/ \mathrm{mm}$ & $11 / 4 \times 40$ \\
Secondary & Casing OD/ID $\times$ length $/ \mathrm{mm}$ & $13 / 11 \times 35$ \\
evaporator & Wick OD/ID $\times$ length $/ \mathrm{mm}$ & $11 / 4 \times 37$ \\
Primary loop & Vapor line OD/ID $\times$ length $/ \mathrm{mm}$ & $3 / 2 \times 700$ \\
& Condenser line OD/ID $\times$ length $/ \mathrm{mm}$ & $2 / 1 \times 700$ \\
& Liquid line OD/ID $\times$ length $/ \mathrm{mm}$ & $3 / 2 \times 600$ \\
Secondary loop & Vapor line OD/ID $\times$ length $/ \mathrm{mm}$ & $3 / 2 \times 30$ \\
& Condenser line OD/ID $\times$ length $/ \mathrm{mm}$ & $2 / 1 \times 260$ \\
Wicks & Liquid line OD/ID $\times$ length $/ \mathrm{mm}$ & $3 / 2 \times 700$ \\
& Porosity $/ ~$ & $55 \%$ \\
Gas reservoir & Minimum capillary radius $/ \mu \mathrm{m}$ & 0.5 \\
\hline
\end{tabular}

and/or secondary evaporators, and its previous stage. It is worth noting that there is a great difference of the maximum capillary pressures for different layout inclination angle at low heat load, while the difference decreases quickly with the heat load and then the maximum capillary pressure tends to almost the same, as shown in Fig. 5.

It is shown in Fig. 4 that the effect of layout inclination angle is mainly manifested assistant/driving or resistant effects of the gravity. When the layout inclination angle is positive, PCC is located above SCC, and the gravity resistant effect is prominent on the working fluid flow in the primary loop. As the driving force, the capillary pressure must be positive and large enough to overcome the total pressure drop including the

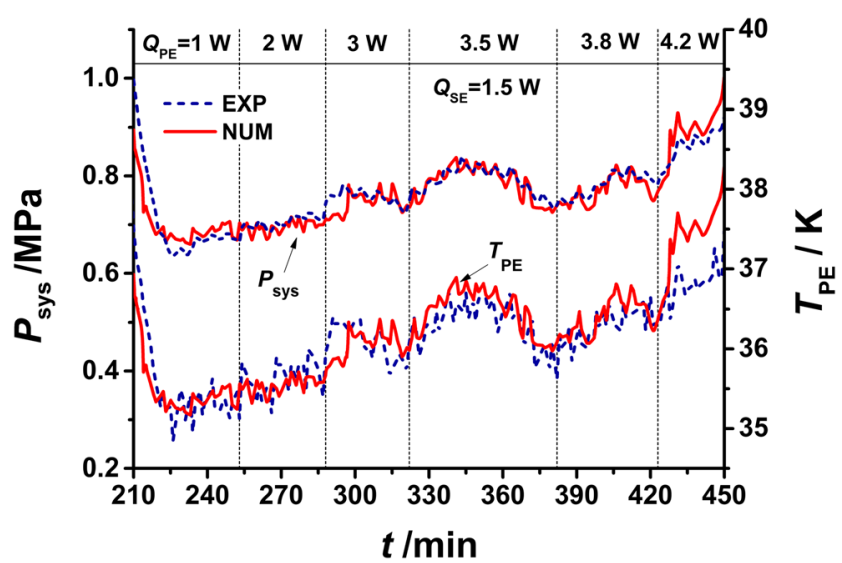

Fig. 2 Comparisons of the numerical simulations of system pressure and operating temperature with the experimental data by Guo et al. (2017) 


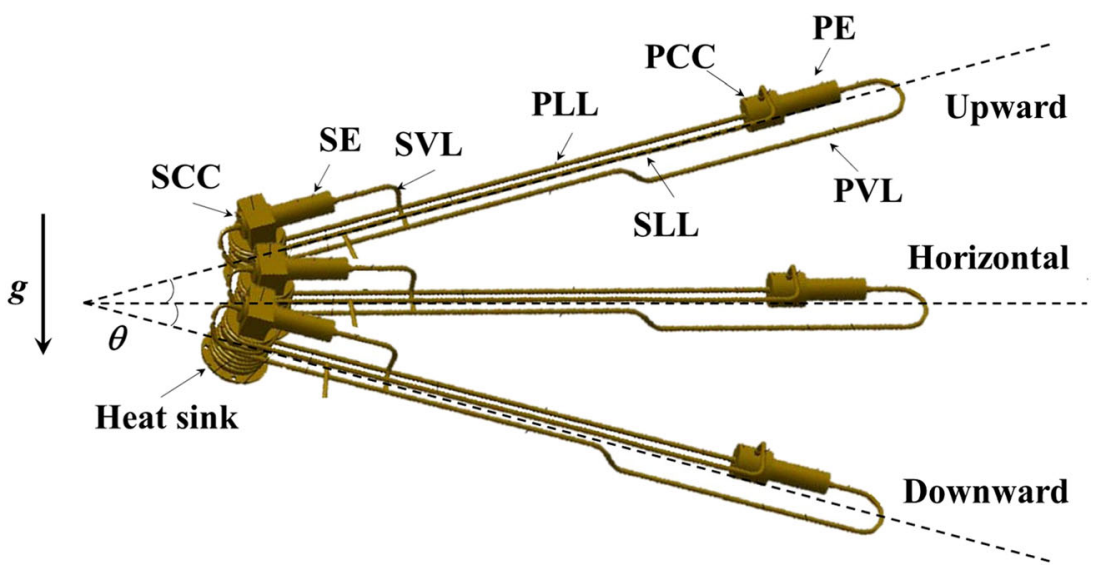

Fig. 3 The schematic of the layout way of facility

gravity term, and then the evaporating interface must be concave. This mode of the system operation is termed here as the normal LHP (n-LHP) mode. Due to the resistant effect of gravity, the primary loop pressure drop increases with the layout inclination angle under n-LHP mode at the same heat load, resulting in a decrease of the heat transport capacity.

On the contrary, when the layout inclination angle is negative, PCC is located below SCC, and then the gravity assistant effect is prominent in the primary loop. There will be three operation modes determined by the primary heat load. The first is the gravity thermosyphon (GTS) mode under lower heat load, in which the difference between the head of gravity and the sum of friction and acceleration pressure drops is greater than the minimum capillary pressure which is determined by the Young-Laplace equation corresponding to the maximum effective radius of the capillary wick, the liquid will then break through the barrier of the capillary pressure, flood the wick and flow into PVL. No stability evaporating interface exists, but nucleation may occur under the input heat from PE. With the increase of the primary heat load, the liquid in the

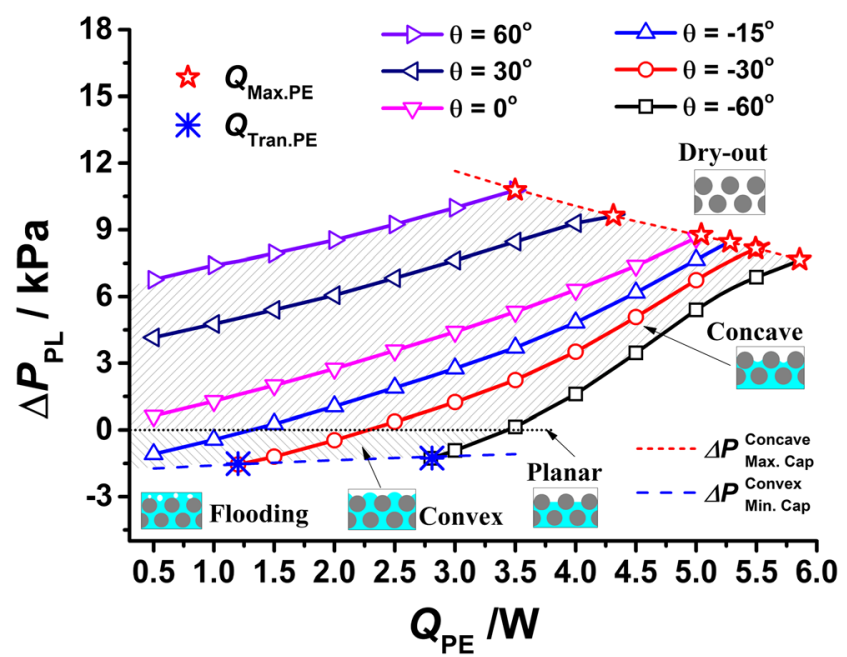

Fig. 4 Variations of the total pressure drop in the primary loop with the primary heat load evaporator groove and vapor chamber of PE will boil away, and then is held inside the capillary wick. The evaporating interface, however, is convex because of the head of gravity is still greater than the sum of friction and acceleration pressure drops in the primary loop. In this mode, the capillary pressure acts as a resistant force, not a driving force, and then is termed here as gravity-driven LHP (g-LHP) mode. The transition heat load $\left(Q_{\text {Tran.PE }}\right)$ from GTS to g-LHP can be determined by the minimums capillary pressure $\left(\Delta P_{\text {Min.Cap }}\right)$. A value of ten times of the minimum effective radius of capillary wick is set for the maximum effective radius of the capillary wick here, and the corresponding minimums capillary pressure is shown in Fig. 4 to determine the transition heat load. With the further increase of the primary heat load, larger flow rate causes larger friction and acceleration pressure drops in the primary loop, exceeding the head of gravity, and then the primary loop will transit to the n-LHP mode from g-LHP. The transition heat load can be determined by the zero position of the sum of the friction, acceleration, and gravity pressure drops in the primary loop. In n-LHP, the evaporating

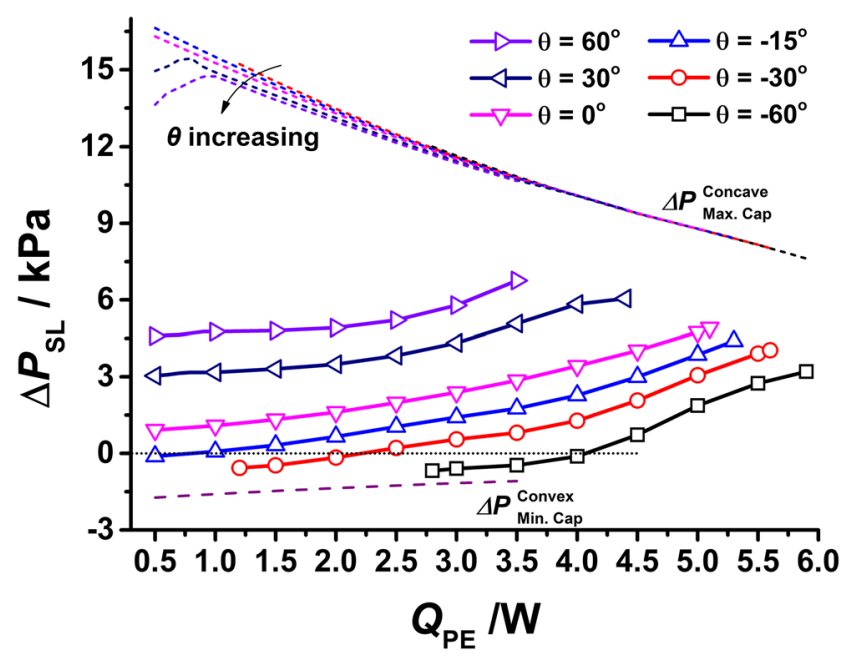

Fig. 5 Variations of the total pressure drop in the secondary loop with the primary heat load 
interface is concave, and the capillary pressure acts as a driving force, and so does the gravity. Therefore, Bai et al. $(2015 \mathrm{a}, \mathrm{b})$ termed it as capillarity-gravity co-driven mode. Only the two LHP modes (g-LHP and n-LHP) are studied here.

Reverse action of gravity in SLL will weaken the gravity effect in the secondary loop, which includes SVL, partial PVL, PC, PLL, PCC, SLL, SC, SCC, and SE. Figure 5 shows the variations of the total pressure drop in the secondary loop $\left(\Delta P_{S L}\right)$ at different layout inclination angles. Comparing with the total pressure drop in the primary loop, it increases slowly in the secondary loop with the primary heat load at constant layout inclination angle. The same trend exists for the increasing layout inclination angle at constant primary evaporator heat load. As shown in Fig. 5, the total pressure drop in the secondary loop always locates in the range between the maximum and minimum capillary pressures in the present study, and then LHP is always maintained in the secondary loop whether the layout inclination angle is positive or negative.

Figure 6 shows the heat transport capacity and the transition heat load between GTP and g-LHP with the layout inclination angle. It is shown that the heat transport capacity is enhanced with the decreasing layout inclination angle due to the weakening of the gravity resistant effect and/or strengthening the gravity assistant effect. In fact, the layout inclination angle affects mainly the change of the pressure drop through the transport lines but slightly the maximum and minimum capillary pressures. It is also easy to find that the larger negative layout inclination angle, the higher transition heat load from g-LHP to GTP, which is in agreement with the Bai et al. (2015a, b).

Figure 7 shows the variations of the operating temperature curves with the primary heat load at different layout inclination angles. The vertical dotted lines mark the corresponding heat transport capacities and the transition heat loads between GTP and g-LHP. When the layout inclination angle is positive, the operating temperature curves show a typical "U" shape, namely the wall temperature of PE decreases firstly and,

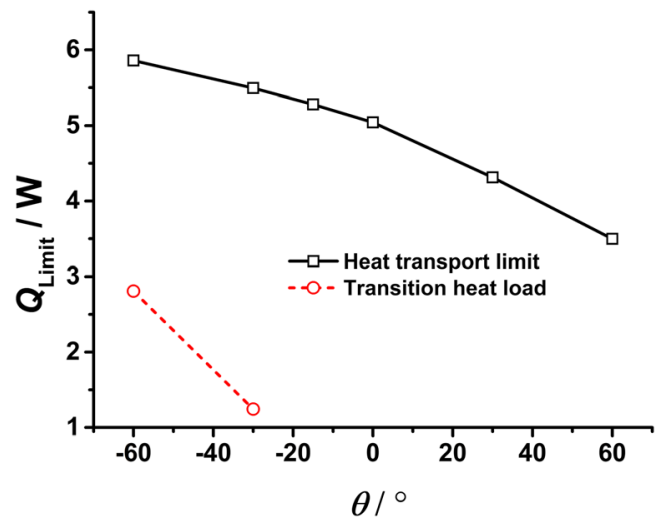

Fig. 6 Variation of the heat transport capacity and the transition heat load between GTP and g-LHP with layout inclination angle

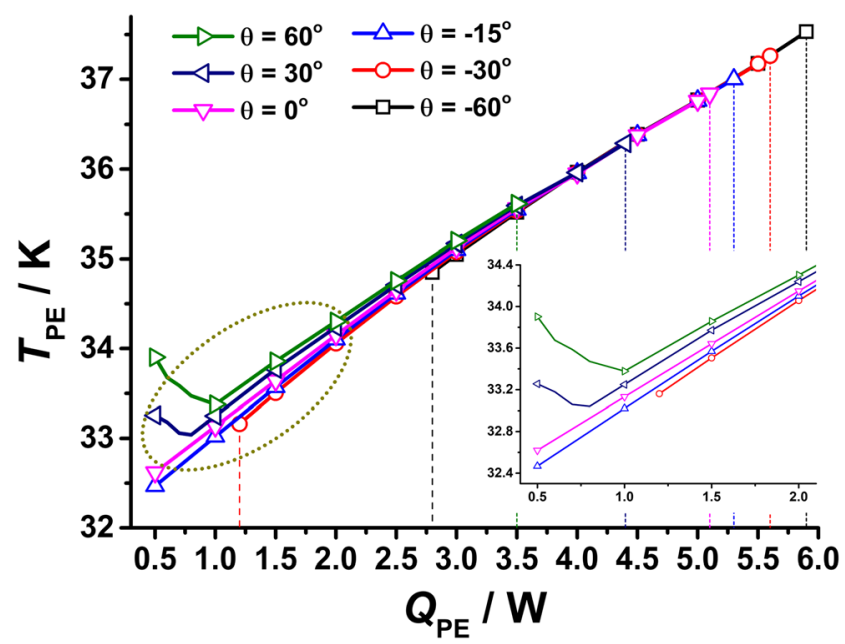

Fig. 7 Variations of the operating temperature with the primary heat load

exceeding a certain value of the primary heat load, increases with the primary evaporator heat load. It is very obvious that the operating temperature decreases with the decreasing layout inclination angle at low primary evaporator heat load, while the difference tends to disappear when the primary heat load is greater than $3.5 \mathrm{~W}$. This trend agrees to the steady numerical simulations by Bai et al. (2010). In the case of horizontal layout, the operating temperature curve is nearly linear, which is similar to the experimental observations by Guo et al. (2018a, b).

Figure 8 shows the effect of the secondary heat loads on the operating temperature in the horizontal layout $\left(\theta=0^{\circ}\right)$. When the secondary heat load is $0.3 \mathrm{~W}$, the operating temperature curve shows a typical "U" shape. With the increase of the secondary heat load, the operating temperature curves move leftward and upward, and the decreasing branch at low primary heat loads will disappear. This trend is similar with the experimental observations by Guo et al. (2018a, b), in which

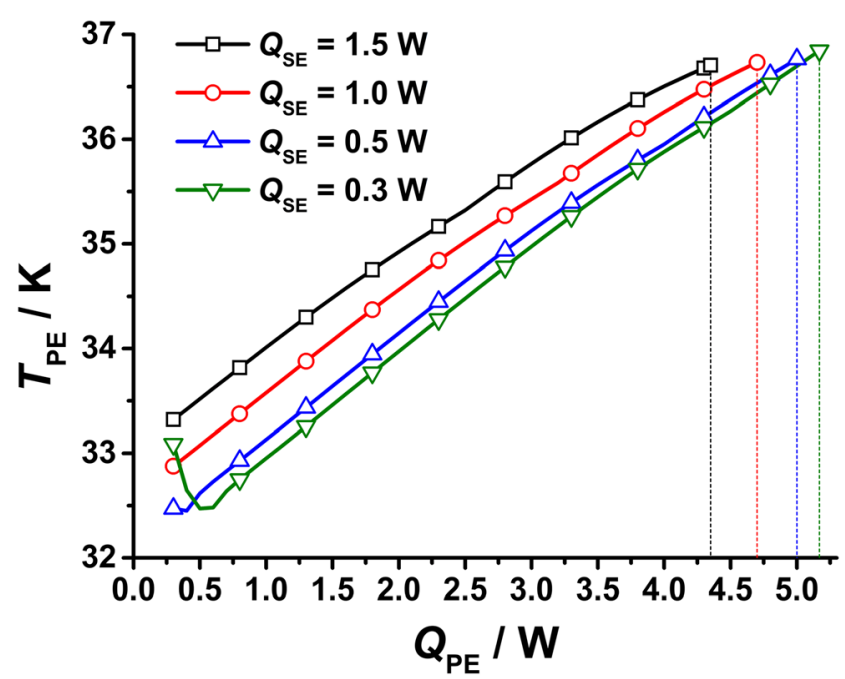

Fig. 8 Variations of the wall temperature of the primary evaporator at different secondary heat loads 


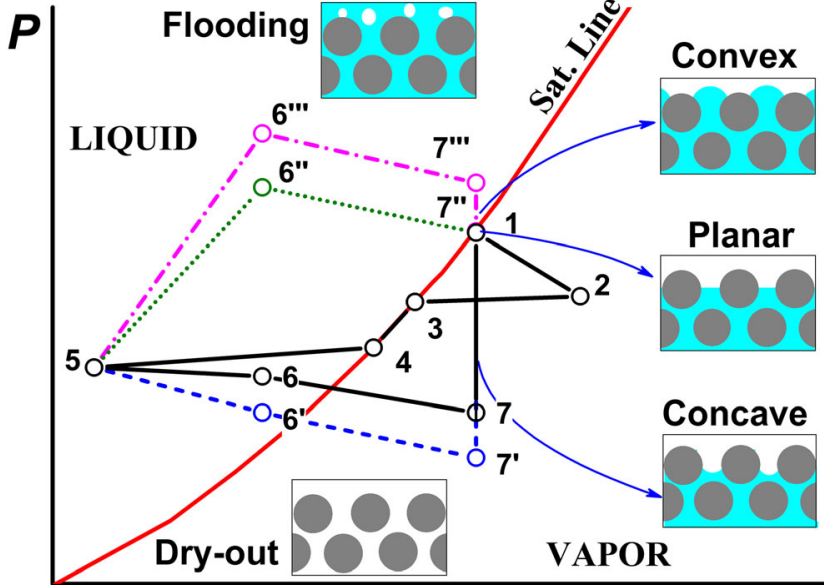

$T$

Fig. 9 Sketch route of thermodynamic state change in the primary loop at different layouts

the operating temperature increases monotonously with the secondary heat load at high primary heat load while sharply decreases at first and then increases with the secondary heat load at low primary heat load.

\section{Discussion}

The gravity effect is usually ignored in the analysis of the route of thermodynamic state change in CLHP. However, the operating process of CLHP exhibits more complicated behaviors with the effect of gravity based on the above simulations, as well as some experimental observations reported in the literature. In order to understand clearly the effect of gravity, Fig. 9 shows in principle the route of the thermodynamic state change in the primary loop.

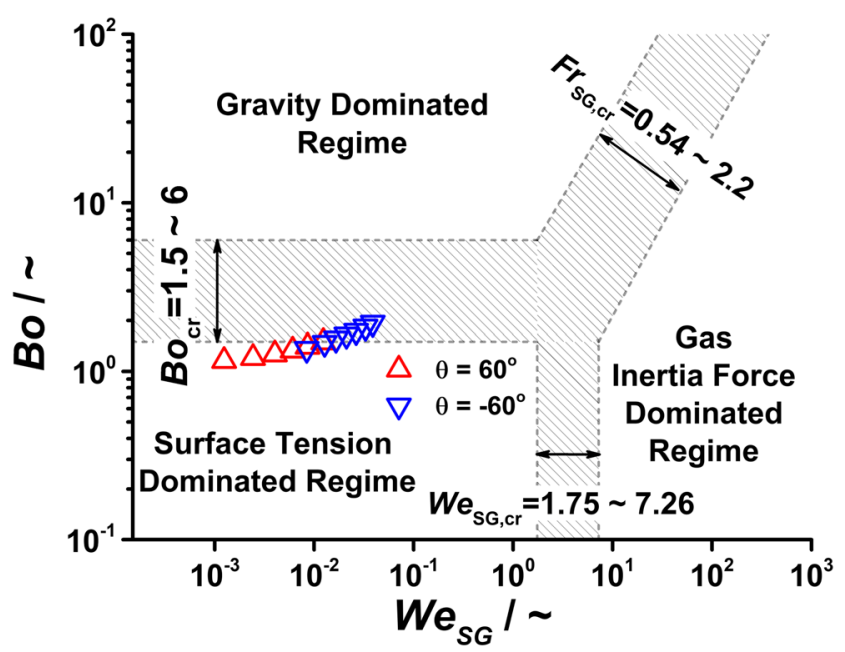

Fig. 10 Typical state of the two-phase vapor-liquid flow at the inlet of PC with different primary heat loads at horizontal layout
At horizontal layout, the CLHP always operates at n-LHP. A stable concave evaporating interface exists, and the capillary force is the driving force. The saturated vapor from the evaporating interface (point 1) flows into PVL (point 2). The pressure decreases due to friction losses along the transport line, and the temperature increases due to the input heat from $\mathrm{PE}$ and/or parasitic heat losses from ambient environment. The resulted superheated vapor flows into PC, releasing sensible and latent heats and being condensed partially into saturation liquid (point 3 ). The single vapor flow then becomes two-phase vapor-liquid flow. Further condensation continually occurs inside PC till no vapor left (point 4). Then the saturated liquid is cooled further to subcooled liquid at the outlet of PC (point 5). The liquid subcooling in the end of PLL (point 6) decreases due to pressure drop and parasitic heat losses. Then the liquid flows into PCC and enters into the capillary wick of PE. Acted by the flow resistance through the capillary wick and the heating from PE, the liquid pressure at the evaporating interface (point 7) is the lowest one while the temperature reaches that of the saturated vapor at the vapor side of the evaporating interface (point 1), resulting in a metastable superheated liquid state. More detail analysis of the route of thermodynamic state change including the secondary loop can be found in He et al. (2018).

At upward layout, a similar route to that at horizontal layout exists with an increasing pressure drop caused by additional gravity pressure drop. Only modified points $6^{\prime}$ and $7^{\prime}$ are shown in Fig. 9, although the actual route should be totally shifted left-up along the saturated line of the working fluid. On the contrary, the actual route for downward layout should be totally shifted right-down along the saturated line. A fixed point for the evaporating interface is shown in Fig. 9 to make more clearly the comparisons among these three cases.

At downward layout where the gravity acts as an assistant or driving force, there are three operating modes, namely $\mathrm{n}$ LHP, g-LHP, and GTP as mentioned above. The first one, in which the metastable superheated liquid state of the working fluid at the liquid side of the evaporating interface locates right below the point 1 but above the point 7, is not shown in Fig. 9 . The capillary pressure still acts as a driving force, and then the evaporating interface remains a concave shape. The n-LHP mode will end when the point $7 "$ overlaps the point 1 . No capillary pressure is needed, resulting in a planar evaporating interface. The liquid at the evaporating interface is in a saturated state. In the g-LHP mode, the evaporating interface becomes convex, and then the capillary pressure acts as a resistance to hold the liquid inside the capillary wick. The liquid at the interface is in a stable subcooled state, which should be heated at first to saturated state and then evaporated by the input heat from PE. The point 7" 'is corresponding to the critical condition related to the transition from g-LHP to GTP. Exceeding the point 7"', the liquid will flood the vapor groove and vapor chamber of PE, and flow into PVL, resulting in the 
transition to GTP. Several kinds of heat and mass transfer ways exist inside PE, including conduction, convection, nucleate boiling, and evaporation, which may result in more complex behaviors, such as an obvious temperature hysteresis caused be nucleate boiling (Nishikawara et al. 2019).

As mentioned above, a basic assumption that the effects of gravity on cross-sectional phase distributions in transport lines and the primary and secondary condensers are ignored is adopted in the present study. Its validation can be found in the frame of dominant force analysis (Du et al. 2018, 2019). Figure 10 shows the comparison of the state of the two-phase vapor-liquid flow at the inlet of PC under different primary heat loads at horizontal layout and the gravity-independence criteria proposed by Zhao et al. (2000). Here, the Bond number (Bo), the Weber number (We) and the Froude number (Fr) are defined as

$$
\left\{\begin{array}{l}
B o=\left(\rho_{L}-\rho_{G}\right) g d^{2} / \sigma \\
W e_{S G}=\rho_{G} U_{S G}^{2} d / \sigma \\
F r_{S G}=U_{S G} / \sqrt{\left(\rho_{L}-\rho_{G}\right) g d / \rho_{G}}=\sqrt{W e_{S G} / B o}
\end{array}\right.
$$

where $\rho, g, d, \sigma$, and $U$ denote respectively the density, gravity, diameter, surface tension, and velocity, while the subscript $L, G$, and $S$ denote liquid, gas and superficial, respectively. Three transition ranges, namely $B o=1.5 \sim 6, F r_{S G}=0.54 \sim 2.2$, and $W e_{S G}=1.75 \sim 7.26$ proposed by Zhao et al. (2000), divide the two-phase flow into three regimes, namely gravity, surface tension, and gas inertia force dominated regimes. The latter two regimes can also be termed as gravity-independent regime. It is evident that the studied two-phase flow falls inside the surface tension dominated regime. That is to say, the crosssectional distribution of the gas and liquid phases can indeed be regarded as gravity-independent in the present study.

\section{Conclusion}

A transient mathematical model was established for study the operation performances of an auxiliary loop type Ne-CLHP. The model validation was demonstrated by comparing the numerical results with the experimental data reported in the literature. The gravity effects caused by different layouts in normal gravity environment on the system operation performance were numerically investigated.

There are three operation modes, namely normal LHP mode (n-LHP), gravity-driven LHP mode ( $\mathrm{g}$-LHP), and gravity thermosyphon mode (GTP), according to different gravity effects. Under the gravity resistance condition with a positive layout inclination angle, the Ne-CLHP is operated in n-LHP. The operating temperature increases with the positive layout inclination angle, and the heat transport capacity decreases. Under the gravity assistance condition with a negative layout inclination angle, all three operation modes may occur according mainly to the primary heat load. Only the two LHP modes are simulated in the present study, which located in the range between the transition heat load from GTP to $\mathrm{g}$ LHP and the heat transport capacity. The lager negative layout incline angle, the higher transition heat load and the heat transport capacity, while lower operating temperature. The results will contribute to the technology research and development, the ground tests and operation control of CLHPs for space applications, as well as the operation control of CLHPs for ground applications.

Acknowledgements The present study is supported financially by the Key Research Program of Frontier Sciences/CAS under the grant of QYZDY-SSW-JSC040, and the National Natural Science Foundation of China (NSFC) under the grants of 11972040 and 51706020.

\section{References}

Bai, L., Lin, G., Wen, D.: Parametric analysis of steady-state operation of a CLHP. Appl. Therm. Eng. 30, 850-858 (2010)

Bai, L., Lin, G., Peterson, G., et al.: Modeling and analysis of supercritical startup of a cryogenic loop heat pipe. J. Heat Transf. 133, 12501 (2011)

Bai, L., Lin, G., Zhang, H., et al.: Experimental study of a nitrogencharged cryogenic loop heat pipe. Cryogenics. 52, 557-563 (2012)

Bai, L., Lin, G., Zhang, H., et al.: Effect of component layout on the operation of a miniature cryogenic loop heat pipe. Int. J. Heat Mass Transf. 60, 61-68 (2013)

Bai, L., Guo, J., Lin, G., et al.: Steady-state modeling and analysis of a loop heat pipe under gravity-assisted operation. Appl. Therm. Eng. 83, 88-97 (2015a)

Bai, L., Zhang, L., Lin, G., et al.: Development of cryogenic loop heat pipes: a review and comparative analysis. Appl. Therm. Eng. 89, 180-191 (2015b)

Brenan, K.E., Campbell, S.L., Petzold, L.R.: Numerical Solution of Initial-Value Problems in Differential Algebraic Equations. SIAM, Philadelphia (1996)

Bugby D., Marland B., Stouffer C., et al. Across-gimbal and miniaturized cryogenic loop heat pipes. Space Technology and Applications International Forum-STAIF, pp. 218-226 (2003)

Bugby, D., Marland, B., Stouffer, C., et al.: Development of advanced tools for cryogenic integration. Adv. Cryog. Eng. 49, 1914-1922 (2004)

Chuang, P.A., Cimbala, J.M., Brenizer, J.S.: Experimental and analytical study of a loop heat pipe at a positive elevation using neutron radiography. Int. J. Therm. Sci. 77, 84-95 (2014)

Du, W.F., Zhao, J.F., Li, K.: Criteria for dominated force regime map in multiphase thermal fluid system. J. Hebei Univ. Water Res. Elec. Eng. 12(1), 1-5 (2018)

Du, W.F., Yue, S.W., Zhao, J.F., et al.: Criteria of gravity-independence in multiphase thermal fluid system. J. Hebei Univ. Water Res. Elec. Eng. (3), 1-7 (2019)

Gully, P., Mo, Q., Yan, T., et al.: Thermal behavior of a cryogenic loop heat pipe for space application. Cryogenics. 51, 420-428 (2011)

Guo, Y., He, J., Lin, G., et al.: Experimental study on the supercritical startup and heat transport capability of a neon-charged cryogenic loop heat pipe. Energy Conversion \& Management. 134, 178-187 (2017)

Guo, Y., Lin, G., Bai, L., et al.: Experimental study of the thermal performance of a neon cryogenic loop heat pipe. Int. J. Heat Mass Transf. 120, 1266-1274 (2018a) 
Guo, Y., Lin, G., Zhang, H., et al.: Investigation on thermal behaviours of a methane charged cryogenic loop heat pipe. Energy. 157, 516-525 (2018b)

He, F.L., Du, W.F., Zhao, J.F., et al.: Transient numerical simulation on cryogenic loop heat pipe. Manned Spaceflight. 84, 512-519 (2018)

He, J., Guo, Y.D., Zhang, H.X., et al.: Design and experimental investigation of a neon cryogenic loop heat pipe. Heat Mass Transf. 53, 3229-3239 (2017)

Hoang T.T., O'Connell T.A., Khrustalev D.K., et al. Cryogenic advanced loop heat pipe in temperature range of $20-30 \mathrm{~K} .12$ th International Heat Pipe Conference, Moscow, Russia (2002)

Hoang, T.T., O'Connell, T.A., Khrustalev, D.K.: Development of a flexible advanced loop heat pipe for across-gimbal cryocooling. Proc. SPIE. 5172, 68-76 (2003)

Kaya, T., Hoang, T.T.: Mathematical modeling of loop heat pipes and experimental validation. J. Thermophys. Heat Transf. 13, 314-320 (1999)

Kaya, T., Perez, R., Gregori, C., et al.: Numerical simulation of transient operation of loop heat pipes. Appl. Therm. Eng. 28, 967-974 (2008)

Launay, S., Sartre, V., Bonjour, J.: Analytical model for characterization of loop heat pipes. J. Thermophys. Heat Transf. 22, 623-631 (2008)

Nishikawara, M., Nagano, H., Kaya, T.: Transient thermo-fluid modeling of loop heat pipes and experimental validation. J. Thermophy. Heat Transfer. 27, 641-647 (2013)
Nishikawara, M., Ueda, Y., Yanada, H.: Static and dynamic liquid-vapor phase distribution in the capillary evaporator of a loop heat pipe. Microgravity Sci.Technol. 31(1), 61-71 (2019)

Okamoto, A., Miyakita, T., Nagano, H.: On-orbit experiment plan of loop heat pipe and the test results of ground test. Microgravity Sci. Technol. 31, 327-337 (2019)

Qu, Z., Chen, G., Zhou, L., et al.: Numerical study on the operating characteristics of cryogenic loop heat pipes based on a onedimensional heat leak model. Energy Conv. Manag. 172, 485-496 (2018)

Yun J., Kroliczek E., Crawford L. Development of a cryogenic loop heat pipe (CLHP) for passive optical bench cooling applications. SAE Paper No. 2002-01-2507 (2002)

Zhao J.F., Xie J.C., Lin H., et al. Experimental study on gas/liquid twophase flow in microgravity. 51st Int. Astronautical Cong., 2-6 October, Rio de Janeiro, Brazil (2000)

Zhao, Y., Yan, T., Liang, J.: Experimental study on a cryogenic loop heat pipe with high heat capacity. Int. J. Heat Mass Transf. 54, 3304 3308 (2011)

Publisher's Note Springer Nature remains neutral with regard to jurisdictional claims in published maps and institutional affiliations. 